Correction to: Some classes of permutation polynomials of the form $b\left(x^{q}+a x+\delta\right)^{\frac{i\left(q^{2}-1\right)}{d}+1}+c\left(x^{q}+a x+\delta\right)^{\frac{j\left(q^{2}-1\right)}{d}+1}+L(x)$ over $\mathbb{F}_{q^{2}}$

\title{
Danyao $\mathrm{Wu}^{1} \cdot$ Pingzhi Yuan ${ }^{2}$
}

Published online: 30 July 2020

(c) Springer-Verlag GmbH Germany, part of Springer Nature 2020

\section{Correction to: Applicable Algebra in Engineering, Communication and Computing https://doi.org/10.1007/s00200-020-00441-z}

In the original publication of the article, the mathematics symbol $q$ was replaced with "s" by mistake while replacing the math mode to text mode. As a result, the first sentence in the following sections are affected and correct sentences should read as given below:

\section{Abstract}

Let $q$ be a prime power and $\mathbb{F}_{q}$ be a finite field with $q$ elements.

\section{Introduction}

Let $\mathbb{F}_{q}$ be the finite field with $q$ elements, where $q$ is a prime power, and let $\mathbb{F}_{q}[x]$ be the ring of polynomials of one variable over $\mathbb{F}_{q}$.

Lemma 2 (See [26]) For a prime power $q$, assume that $a \in \mathbb{F}_{q^{2}}$ with $a^{q+1}=1$ and $g \in \mathbb{F}_{q^{2}}$ is a primitive element of $\mathbb{F}_{q^{2}}$.

The original article can be found online at https://doi.org/10.1007/s00200-020-00441-z.

Danyao $\mathrm{Wu}$

wudanyao111@163.com

Pingzhi Yuan

yuanpz@scnu.edu.cn

1 School of Computer Science and Technology, Dongguan University of Technology, Dongguan 523808, China

2 School of Mathematics, South China Normal University, Guangzhou 510631, China 
Theorem 1 For a prime power $q$ and positive integers $d, i, j$ with $q \equiv 1(\bmod d)$, assume that $b, c \in \mathbb{F}_{q}$ and $a, \delta \in \mathbb{F}_{q^{2}}$ with $a^{1+q}=1$.

Theorem 2 For a prime power $q$ and positive integers $d, i, j$ with $q \equiv 1(\bmod d)$, assume that $b, c \in \mathbb{F}_{q}, a \in \mathbb{F}_{q^{2}}$ with $a^{1+q}=1$, and $g \in \mathbb{F}_{q^{2}}$ is a primitive element of $\mathbb{F}_{q^{2}}$.

Theorem 3 For a prime power $q$ and positive integers $d, i, j, s, k$ with $q \equiv-1(\bmod d), i q \equiv s(\bmod d)$ and $j q \equiv k(\bmod d)$, assume that $b, c \in \mathbb{F}_{q}$ and $a, \delta \in \mathbb{F}_{q^{2}}$ satisfying $a^{1+q}=1$.

The original article has been updated accordingly.

Publisher's Note Springer Nature remains neutral with regard to jurisdictional claims in published maps and institutional affiliations. 